\title{
Urinary sediment microRNAs can be used as potential noninvasive biomarkers for diagnosis, reflecting the severity and prognosis of diabetic nephropathy
}

\author{
Qiuxia Han ${ }^{1,2,4}$, Youcai Zhang ${ }^{3,4}$, Tingting $\mathrm{Jiao}^{3}, \mathrm{Qi} \mathrm{Li}^{2}$, Xiaonan Ding ${ }^{2}$, Dong Zhang ${ }^{1,2}$, Guangyan Cai ${ }^{1,2}$ and Hanyu Zhu (iD ${ }^{2 凶}$
}

(c) The Author(s) 2021

BACKGROUND: Patients with both diabetes mellitus (DM) and kidney disease could have diabetic nephropathy (DN) or nondiabetic renal disease (NDRD). IgA nephropathy (IgAN) and membranous nephropathy (MN) are the major types of NDRD. No ideal noninvasive diagnostic model exists for differentiating them. Our study sought to construct diagnostic models for these diseases and to identify noninvasive biomarkers that can reflect the severity and prognosis of DN.

METHODS: The diagnostic models were constructed using logistic regression analysis and were validated in an external cohort by receiver operating characteristic curve analysis method. The associations between these microRNAs and disease severity and prognosis were explored using Pearson correlation analysis, Cox regression, Kaplan-Meier survival curves, and log-rank tests. RESULTS: Our diagnostic models showed that miR-95-3p, miR-185-5p, miR-1246, and miR-631 could serve as simple and noninvasive tools to distinguish patients with DM, DN, DM with IgAN, and DM with MN. The areas under the curve of the diagnostic models for the four diseases were $0.995,0.863,0.859$, and 0.792 , respectively. The miR-95-3p level was positively correlated with the estimated glomerular filtration rate $(p<0.001)$ but was negatively correlated with serum creatinine $(p<0.01)$, classes of glomerular lesions $(p<0.05)$, and scores of interstitial and vascular lesions $(p<0.05)$. However, the miR-631 level was positively correlated with proteinuria $(p<0.001)$. A low miR-95-3p level and a high miR-631 level increased the risk of progression to end-stage renal disease $(p=0.002, p=0.011)$.

CONCLUSIONS: These four microRNAs could be noninvasive tools for distinguishing patients with DN and NDRD. The levels of miR$95-3 p$ and miR-631 could reflect the severity and prognosis of DN.

Nutrition and Diabetes (2021)11:24; https://doi.org/10.1038/s41387-021-00166-z

\section{INTRODUCTION}

The incidence of diabetes mellitus (DM) has gradually increased with the development of a social economy, and the number of patients with diabetes is expected to exceed 693 million by 2045 [1]. Approximately $40 \%$ of diabetic patients are prone to the development of diabetic nephropathy (DN) [2-4]. DN is one of the most common microvascular complications of diabetes and is considered the most common cause of end-stage renal disease (ESRD) $[5,6]$. Many scholars have found that some patients with diabetes and kidney disease have different clinical manifestations and sensitivities to treatment than those with typical DN [7, 8]. Apart from DN, diabetic patients with kidney disease may also have non-diabetic renal disease (NDRD) $[9,10]$. According to reports, the incidence of NDRD is $\sim 53 \%$, which varies among countries, possibly due to race, region, and selection criteria for renal biopsy $[10,11] \lg A$ nephropathy (IgAN) and membranous nephropathy (MN) are the major types of NDRD [11, 12]. Li et al. [13] conducted a 5year longitudinal follow-up survival analysis and found that the prognosis of the NDRD group was better than that of the DN group, which indicates that patients with type 2 diabetes who were diagnosed with NDRD at the early stage can achieve an improved prognosis after appropriate and timely treatment measures are given. For diabetic patients with kidney disease, it is not feasible to estimate a diagnosis of DN based on experience $[9,14]$. Instead, consideration should be given to diabetic patients with other kidney diseases, especially MN and IgAN, which are very common [15]. Since DN and NDRD are not the same type of disease, their pathological characteristics, clinical manifestations, treatment responses, disease progression rates, and prognoses all differ, and thus, an accurate differential diagnosis is particularly important [16]. The gold standard for distinguishing DN and NDRD is renal biopsy, but it cannot be performed routinely because of its invasiveness,

\footnotetext{
${ }^{1}$ School of Medicine, Nankai University, State Key Laboratory of Kidney Diseases, National Clinical Research Center of Kidney Diseases, First Medical Center of Chinese PLA General Hospital, Tianjin, China. ${ }^{2}$ Department of Nephrology, First Medical Center of Chinese People's Liberation Army General Hospital, Nephrology Institute of the Chinese People's Liberation Army, Beijing Key Laboratory of Kidney Disease Research, Beijing, China. ${ }^{3}$ Department of Nephrology, Jiaozuo People's Hospital, Jiaozuo, China. ${ }^{4}$ These authors

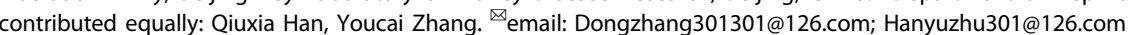

Received: 18 August 2020 Revised: 20 May 2021 Accepted: 1 June 2021

Published online: 30 June 2021 
high cost, and high technical requirements. Therefore, a simple, stable, and reliable identification method is an urgent clinical need. However, an ideal noninvasive diagnostic model for DN has not yet been established nationally or abroad.

MicroRNA is an endogenous non-coding single-stranded RNA consisting of 21-25 nucleotides [17]. MicroRNAs can induce the degradation of messenger RNAs and block protein translation. They also play a very important regulatory role in the occurrence and development of kidney disease [17]. Therefore, the discovery of blood- and urine-specific microRNAs in patients with DN has provided a new direction for identifying diagnostic biomarkers for DN and has enabled the timely screening and disease monitoring of DN [18]. Many studies have shown that the urine of patients with DN contains numerous exfoliated podocytes, the number of which is proportional to the severity of proteinuria [19]. Podocyte shedding is an important pathological feature of DN, and proteinuria is an important clinical feature of DN [20]. Given that urinary sediment cells are mainly derived from the kidney, detection of biomarkers from the urinary sediment of patients can more accurately capture the specific biological information changes that occur in kidney disease [21]. In addition, urinary sediment is obtained by the centrifugation of urine, this noninvasive and easy method can contribute to early screening and disease monitoring [22].

Although some studies have focused on biomarkers in blood and urine supernatants from patients with DN, no studies have investigated the role of microRNAs in the diagnosis of DN based on urinary sediment. Compared with blood sample collection, urine sample collection is more convenient, less invasive, and painless. Urinary sediment has a higher nucleic acid content and is relatively less affected by humoral metabolic factors than urine supernatant [23]. Therefore, urinary sediment is an ideal specimen for identifying noninvasive diagnostic biomarkers.

The purpose of this study was to investigate microRNA alterations among DM, DN, and NDRD and construct diagnostic models for these diseases, and to identify noninvasive biomarkers that can reflect the severity and prognosis of DN.

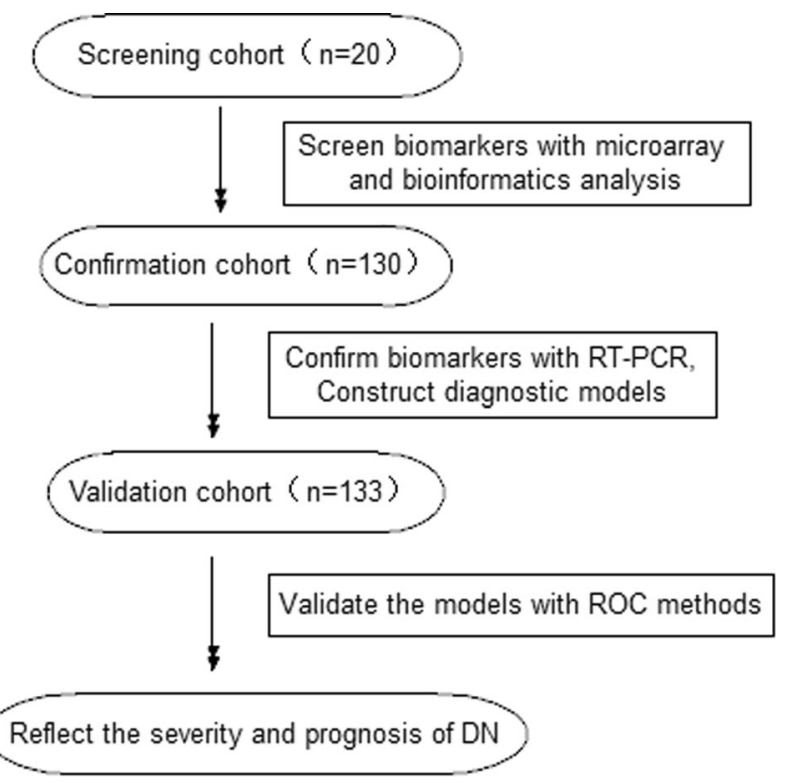

Fig. 1 The design scheme for constructing and identifying the diagnostic models in this study. PCR polymerase chain reaction, ROC receiver operating characteristic, DN diabetic nephropathy.

\section{MATERIALS AND METHODS}

\section{Study design and subject enrollment}

In all, 283 eligible subjects at the General Hospital of the Chinese People's Liberation Army were enrolled from January 2017 to September 2019. The study design scheme is illustrated in Fig. 1. This study was conducted in accordance with the Declaration of Helsinki for research involving human subjects and was approved by the Ethics Committee of the Chinese PLA General Hospital (No. S2014-012-01). Written informed consent for inclusion was obtained from each participant.

A diagnosis of type 2 diabetes was defined according to either the fasting plasma glucose (FPG) level or 2-h plasma glucose level after a $75 \mathrm{~g}$ oral glucose tolerance test (OGTT) or according to the hemoglobin A1c (HbA1c) based on the American Diabetes Association (ADA) criteria [24]. The pathological classification of DN was performed according to the Renal Pathology Society (RPS) classification system [25]. Indications for renal biopsy were matched with the criteria for NDRD in the 2007 Kidney Disease Outcomes Quality Initiative (KDOQI) guidelines [26]. As for the sample size calculation, when we set the confidence level as 0.95 , the power is 0.8 , the distance from mean to limits is 0.3 , standard deviation is 0.4 , the interval type is two-sided, the required sample size is 17 .

The inclusion criteria were as follows: diagnosis of type 2 diabetes; pathological diagnosis of DN or NDRD; age over 18 years; underwent renal biopsy at our hospital; agreed to join this experimental study and voluntarily signed an informed consent form. The exclusion criteria were as follows: patients with an incomplete or unclear medical history; combined urinary tract infection, immune system disease, malignant tumor or pregnancy; a confirmed diagnosis of renal disease before a type 2 diabetes diagnosis; a clinically confirmed diagnosis of NDRD, including lupus nephritis and Henoch-Schönlein purpura nephritis; and history of familial hereditary nephropathies. To evaluate whether microRNAs in urinary sediment may be potential biomarkers that can differentiate DN from NDRD, patients with other kidney diseases accompanied by DN or NDRD and those with a pathological diagnosis of DN combined with NDRD were excluded from this study. The kidney tissues were obtained by percutaneous renal biopsy. Sections were routinely stained by hematoxylin and eosin (H\&E), periodic acid-Schiff (PAS), and periodic acid silver methenamine (PASM). The pathological findings were independently evaluated by two authoritative pathologists.

\section{Urine collection and RNA extraction}

Morning urine specimens of $150-250 \mathrm{ml}$ were collected from DN and NDRD patients before renal biopsy and from DM patients and were immediately centrifuged at $3000 \times g$ at $4{ }^{\circ} \mathrm{C}$ for $30 \mathrm{~min}$ and at $13,000 \times g$ at $4{ }^{\circ} \mathrm{C}$ for $5 \mathrm{~min}$. Then, the supernatant was discarded, and urinary sediment was retained. TRIzol (Invitrogen, USA) was used to extract total RNA from urinary sediment according to the manufacturer's protocol. The concentration and purity of the total RNA were measured with a NanoDrop 2000 spectrophotometer (Thermo Scientific, USA).

\section{MicroRNA microarray analysis}

Human microRNA microarrays from Agilent Technologies $\left(8^{*} 60 \mathrm{~K}\right)$ containing probes for 2549 human microRNAs from the miRBase V21.0 database were used. The microarray image information was converted into spot intensity values using Scanner Control Software Rev. 7.0 (Agilent Technologies, Santa Clara, CA). Raw data were normalized by Quantile algorithm, included in the $\mathrm{R}$ package AgiMicroRna [27]. The microarray experiments were performed at Shanghai Biotechnology Corporation and microRNAs with altered expression levels were screened in each experimental group according to the manufacturer's protocol. The screening cohort included 5 type 2 DM patients, 6 DN patients, and 9 NDRD (4 lgAN and $5 \mathrm{MN}$ ) patients.

\section{Reverse transcription and quantitative real-time PCR}

Total RNA ( $800 \mathrm{ng}$ ) was used to perform CDNA synthesis using the miRcute plus microRNA First-strand cDNA Synthesis Kit (catalog number KR211-02; Tiangen Biotechnology Co., Ltd., Beijing) according to the manufacturer's instructions. PCR on the samples was performed using a miRcute plus microRNA qPCR Detection Kit (catalog number FP401; Tiangen Biotechnology Co., Ltd., Beijing) and an Applied Biosystems 7500 Real-Time PCR System (Applied Biosystems, USA) according to the manufacturer's instructions. All PCR experiments were performed in triplicate and were followed by melting curve analysis to verify specificity and identity of the qPCR products. All primers were purchased from Tiangen Biotechnology 
Table 1. Comparison of urinary sediment microRNA expression profiles among different groups in the confirmation cohort.

\begin{tabular}{|c|c|c|c|c|}
\hline & miR-95-3p & miR-185-5p & miR-1246 & miR-631 \\
\hline DM Group & $0.246 \pm 0.141^{* * *}$ & $0.467 \pm 0.178^{* * *}$ & $0.421 \pm 0.161^{* * *}$ & $0.527 \pm 0.208^{* * *}$ \\
\hline DN Group & $0.814 \pm 0.380$ & $0.828 \pm 0.203$ & $0.891 \pm 0.217$ & $0.843 \pm 0.243$ \\
\hline IgAN Group & $0.671 \pm 0.169^{*}$ & $1.051 \pm 0.269^{* *}$ & $1.017 \pm 0.283^{*}$ & $0.728 \pm 0.178^{* *}$ \\
\hline MN Group & $0.375 \pm 0.119^{* * *}$ & $0.953 \pm 0.278^{*}$ & $0.721 \pm 0.201^{* *}$ & $0.608 \pm 0.174^{* *}$ \\
\hline
\end{tabular}

$D M$ diabetes mellitus (type 2), $D N$ diabetic nephropathy, $\lg A N \lg A$ nephropathy, $M N$ membranous nephropathy.

${ }^{*}$ Other groups compared with DN group, ${ }^{*} p<0.05 ;{ }^{* *} p<0.01$; and ${ }^{* * *} p<0.001$.

Company (Beijing); U6 served as the endogenous reference control, and the relative microRNA expression levels were expressed as $2^{-\Delta \Delta C T}$

\section{Statistical methods}

Normally distributed data were expressed as the mean \pm standard deviation (SD) and were compared using unpaired Student's $t$-tests. Nonnormally distributed data were expressed as medians with the corresponding 25th and 75th percentiles (interquartile range) and were compared using Mann-Whitney U-tests. All tests are two-sided. Values of the relative microRNA expression levels that were out of the average background \pm 2 standard deviations (SDs) were removed from each data point to minimize possible systematic variation. Four diagnostic models of type 2 DM (Model DM), DN (Model DN), NDRD-IgAN (Model IgAN), and NDRD-MN (Model MN) were constructed according to the relative microRNA expression levels based on a forward stepwise logistic regression analysis. The diagnostic performance of the diagnostic models was evaluated by receiver operating characteristic (ROC) curve analysis. Pearson correlation was used to test associations between relative microRNA expression levels and clinical and pathological parameters related to the severity of DN. Cox regression, Kaplan-Meier survival curves, and the log-rank test were performed to analyze dialysis-free survival. $P$ values $<0.05$ were considered statistically significant. Statistical analyses were performed using SPSS statistics 21.0 software (version 21.0 SPSS, Chicago, IL, USA) and GraphPad Prism software (Vision 8, San Diego, (A, USA).

\section{RESULTS}

MicroRNA microarray analysis in the screening phase

MicroRNA microarrays were used to analyze the global expression profiles of the four groups (DM, DN, IgAN, and MN). The microRNA profiling of global urinary sediment from patients in the different groups is shown in Supplementary Fig. S1. The volcano plots of microRNAs evaluated in the screening phase are reported in Supplementary Figs. S2-S7. We selected microRNAs with fold changes $>2$ or $<0.5$ and $p$ values $<0.05$. The total numbers of microRNAs evaluated in the screening phase are reported in Supplementary Tab. S1. We then analyzed the data combined with the target gene prediction websites and biological information analysis. Finally, the sample size was expanded for verification.

\section{Confirmation of the candidate microRNAs and construction of the diagnostic models}

In all, 130 participants were enrolled in the confirmation cohort. The baseline characteristics of the subjects are shown in Supplementary Tab. S2. A comparison of urinary sediment microRNA expression profiles among the different groups in the confirmation cohort is shown in Table 1. The expression levels of four microRNAs were different in the DN group versus all other groups. The relative expression levels of miR-95-3p and miR-631 were increased in the DN group compared with the DM group (both $p<0.001), \operatorname{lgAN}$ group $(p<0.05$ and $p<0.01)$, and MN group $(p<0.001$ and $p<0.01)$. The miR-185-5p level was increased in the DN group compared with the DM group $(p<0.001)$ but was decreased compared with the IgAN group $(p<0.01)$ and $\mathrm{MN}$ group $(p<0.05)$. The miR-1246 level was increased in the DN
Table 2. Evaluation parameters of the diagnostic model based on the logistics regression results.

\begin{tabular}{llll} 
Model & $\begin{array}{l}\text { Positive } \\
\text { predictive value }\end{array}$ & $\begin{array}{l}\text { Negative } \\
\text { predictive value }\end{array}$ & Accuracy \\
\hline Model DM & 0.804 & 0.976 & 0.910 \\
\hline Model DN & 0.725 & 0.951 & 0.865 \\
\hline Model IgA & 0.778 & 0.904 & 0.887 \\
\hline Model MN & 0.818 & 0.877 & 0.872
\end{tabular}

$D M$ diabetes mellitus, $D N$ diabetic nephropathy, $\lg A N \lg A$ nephropathy, $M N$ membranous nephropathy.

group compared with the DM group $(p<0.001)$ and the MN group $(p<0.01)$ but was decreased compared with the IgAN group $(p<0.05)$.

We then used logistics regression to establish a diagnostic model for $\mathrm{DM}, \mathrm{DN}, \lg \mathrm{AN}$, and $\mathrm{MN}$ based on the microRNA expression level in the confirmation cohort.

The DM diagnostic model is as follows:

Model DM $=1 / 1+\mathrm{e}^{-(21.536-19.775 \times \mathrm{miR}-185-5 \mathrm{p}-15.082 \times \mathrm{miR}-1246)}$

The DN diagnostic model is as follows:

Model $\mathrm{DN}=1 / 1+\mathrm{e}^{-(-5.507+3.973 \times \mathrm{miR}-95-3 \mathrm{p}+3.773 \times \mathrm{miR}-631)}$

The IgAN diagnostic model is as follows:

Model $\lg A N=1 / 1+\mathrm{e}^{-(-8.031+3.453 \times \mathrm{miR}-185-5 \mathrm{p}+3.846 \times \mathrm{miR}-1246)}$

The MN diagnostic model is as follows:

Model $\mathrm{MN}=1 / 1+\mathrm{e}^{-(-2.328-2.297 \times \mathrm{miR}-95-3 p+4.083 \times \mathrm{miR}-185-5 p}-2.279 \times \mathrm{miR}-$

Evaluation parameters of the diagnostic model are shown in Table 2 . The accuracies of our four models are $0.910,0.865,0.887$, and 0.872 . These parameters showed that our model is reliable. In addition, we tested the diagnostic model using the ROC method based on the results (predicted probability) of logistic regression (Fig. 2). The areas under the curve of these four models are 0.995, $0.863,0.859$, and 0.792. These data further show that the diagnostic model is reliable (Supplementary Tab. S3).

\section{Validation of the diagnostic models}

The constructed models based on the confirmation cohort were then applied to the validation cohort of 133 patients to evaluate their diagnostic power. As shown in Fig. 3, all candidate microRNAs were generally coincident with the results in the confirmation cohort. The details of the ROC analyses of the constructed models are shown in Supplementary Tab. S3.

The ROC curves indicated that the Model DM ( $A U C=0.928)$ had the highest diagnostic accuracy for distinguishing DM from the other three diseases (Fig. 3a). The ROC curves indicated that the Model DN (AUC $=0.844$ ) had high diagnostic accuracy for distinguishing DN from the other three diseases (Fig. 3b). Moreover, the ROC curves indicated that the Model IgAN (AUC 

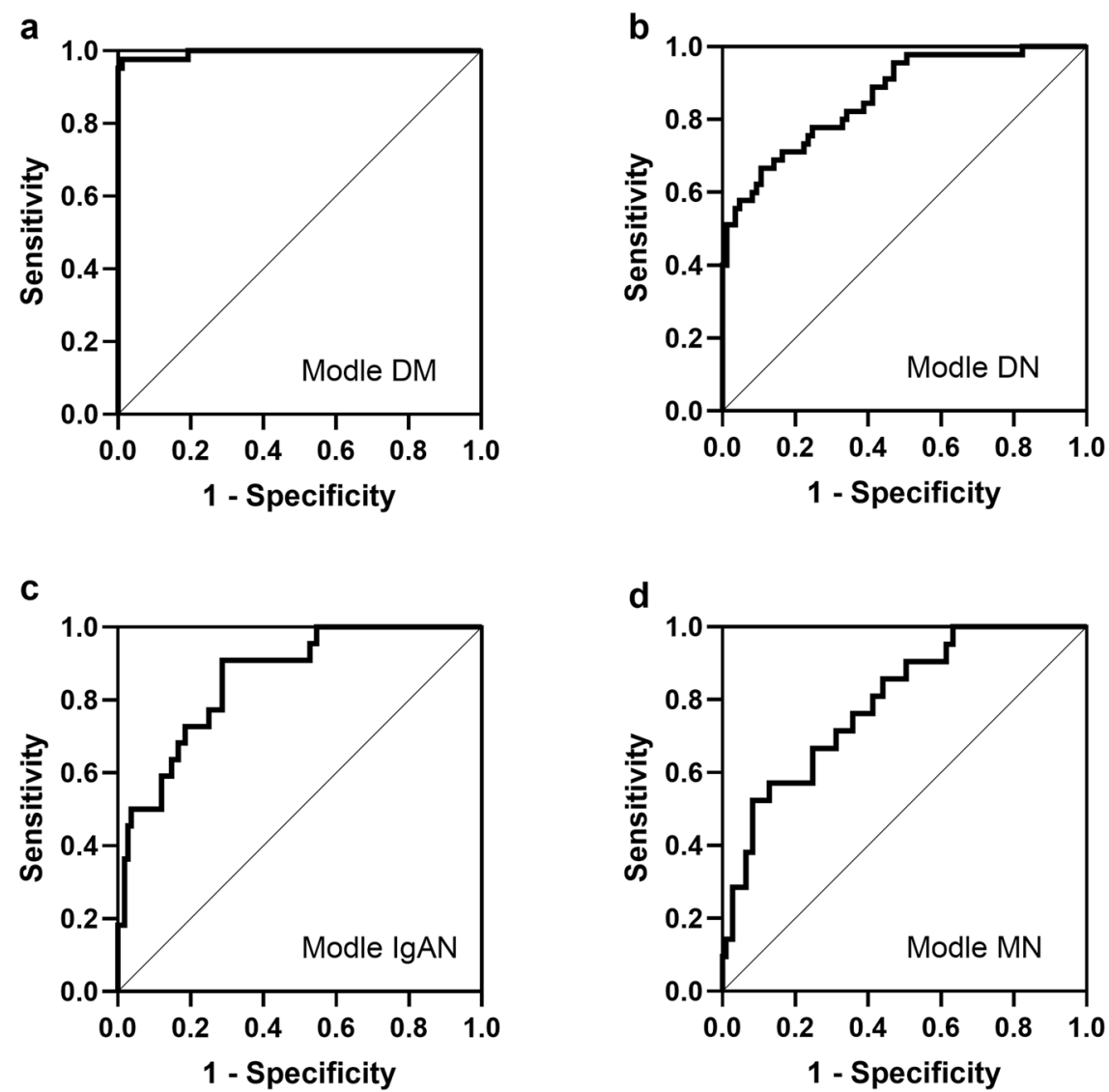

Fig. 2 The diagnostic accuracy of the diagnostic models was determined by ROC analysis in the confirmation cohort. a-d The ROC analysis is shown for Model DM, Model DN, Model IgAN, and Model MN in the confirmation cohort. DM diabetes mellitus, DN diabetic nephropathy, IgAN IgA nephropathy, MN membranous nephropathy.

$=0.849)$ had high diagnostic power for distinguishing IgAN from the other three diseases (Fig. 3c). The ROC curves indicated that the Model MN (AUC $=0.761)$ had moderate diagnostic power for distinguishing MN from the other three diseases (Fig. 3d).

\section{Correlations between relative microRNA expression levels and severity of DN}

To investigate the correlations between relative microRNA expression levels and the severity of DN, we performed a Pearson correlation analysis of microRNA expression levels and clinical and pathological parameters related to the severity of DN (Table 3). The level of miR-95-3p was positively correlated with the estimated glomerular filtration rate (eGFR) $(p<0.001)$ but was negatively correlated with serum creatinine $(p<0.01)$, classes of glomerular lesions $(p<0.05)$, and scores of interstitial and vascular lesions $(p<0.05)$. The level of miR-631 was positively correlated with proteinuria $(p<0.001)$, whereas the levels of miR-185-5p and miR-1246 showed no correlation with any clinical and pathological parameters related to the severity of DN.

\section{Association of microRNAs and dialysis-free survival of DN patients}

To investigate the effects of these microRNAs on the dialysis-free survival of DN patients, we performed a multivariate Cox regression analysis (Table 4). A higher miR-95-3p level decreased the risk of progression to ESRD and treatment with dialysis $(p=0.016$, odds ratio $(\mathrm{OR})=0.305)$. However, a higher miR-631 level increased the risk of progression to ESRD and treatment with dialysis $(p=0.013, \mathrm{OR}=7.890)$. To further explore the effects of miR-95-3p and miR-631 on the dialysis-free survival of DN patients, we performed a Kaplan-Meier analysis (Fig. 4). The subjects were dichotomized based on the mean of the covariates $(0.822$ for miR95-3p and 0.938 for miR-631). A high level of miR-95-3p decreased the risk of progression to ESRD (Fig. $4 a, p=0.002$ ), while a high level of miR-631 increased the risk of progression to ESRD (Fig. $4 \mathrm{~b}$, $p=0.011)$.

\section{DISCUSSION}

In recent years, the incidence of diabetes has continued to increase, which has resulted in a heavy economic burden on society [28]. Diabetic patients with kidney disease could have DN or NDRD $[9,10]$. Compared with NDRD, the treatment effect and prognosis of DN are worse than those of NDRD, and the pathological course is difficult to reverse [29]. The diagnostic gold standard for DN and NDRD is renal biopsy, which is an invasive procedure that has not been widely applied due to its high cost and high technical requirements $[6,30]$. Therefore, the discovery of new noninvasive methods that can identify DN and NDRD has important clinical value. MicroRNAs have become biomarkers of many types of diseases due to their function and varied biological effects [17, 31, 32].

In the present study, microarray analysis was used to identify differentially expressed microRNAs in urinary sediment samples from DM, DN, and NDRD (IgAN and MN) patients. To expand the sample size for verification, many more urine samples were collected to confirm the candidate microRNAs and to construct diagnostic models in the confirmation cohort. We found that the levels of miR-95-3p, miR-185-5p, miR-1246, and miR-631 were all statistically significant in the DN group compared with the other groups. The diagnostic models we established had high diagnostic 
a

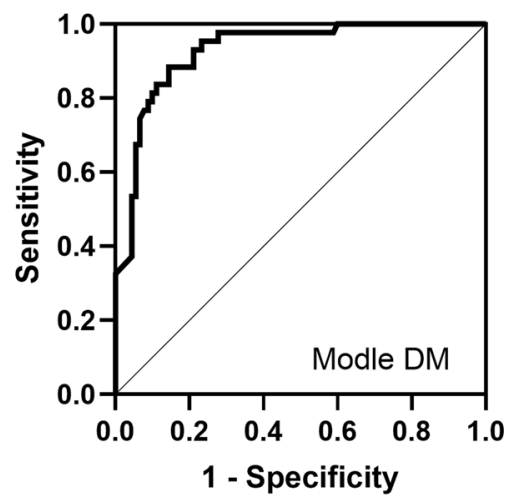

C

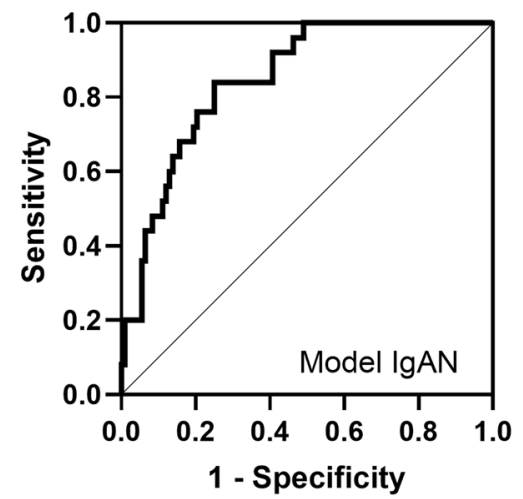

b

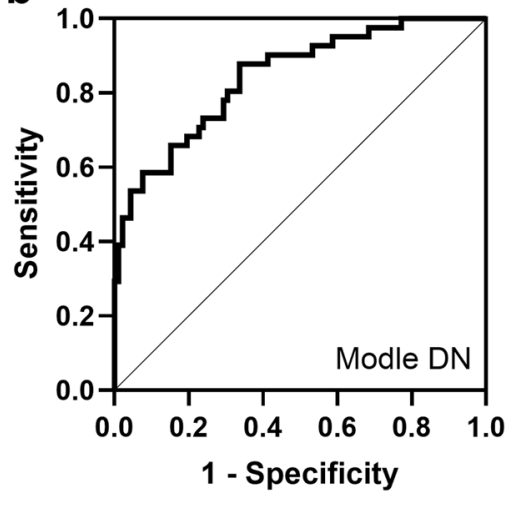

d

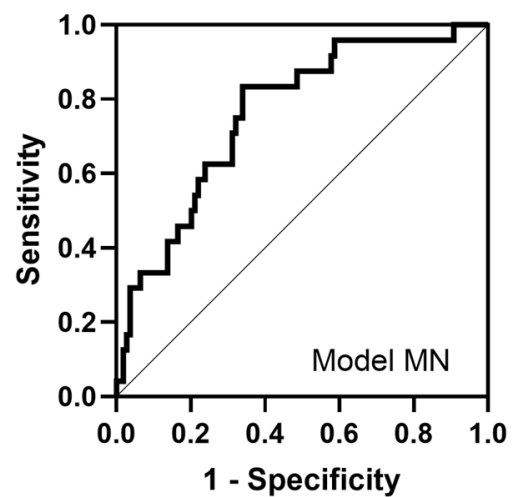

Fig. 3 The diagnostic accuracy of the diagnostic models was determined by ROC analysis in the validation cohort. a-d The ROC analysis is shown for Model DM, Model DN, Model IgAN, and Model MN in the validation cohort. DM diabetes mellitus, DN diabetic nephropathy, IgAN IgA nephropathy, MN membranous nephropathy.

Table 3. Pearson correlations of relative microRNA expression levels and clinical parameters related to the severity of diabetic nephropathy

\begin{tabular}{lllll} 
& miR-95-3p & miR-185-5p & miR-1246 & miR-631 \\
\hline Proteinuria $(\mathrm{g} / 24 \mathrm{~h})$ & -0.184 & 0.084 & 0.055 & $0.532^{* * *}$ \\
\hline eGFR $\left(\mathrm{ml} / \mathrm{min} / 1.73 \mathrm{~m}^{2}\right)$ & $0.429^{* * *}$ & 0.039 & 0.078 \\
\hline Serum creatinine & $-0.369^{* *}$ & -0.161 & -0.062 & -0.143 \\
\hline Classes of glomerular lesions & $-0.280^{*}$ & -0.096 & 0.001 & -0.115 \\
\hline Scores of Interstitial and vascular lesions & $-0.297^{*}$ & -0.053 & -0.187
\end{tabular}

The estimated glomerular filtration rate (eGFR) was calculated using the Chronic Kidney Disease Epidemiology Collaboration (CKD-EPI) equation. ${ }^{*} p<0.05 ;{ }^{* *} p<0.01$; and ${ }^{* * *} p<0.001$.

Table 4. Multivariate Cox regression analysis for dialysis-free survival of patients with diabetic nephropathy.

\begin{tabular}{|c|c|c|c|c|c|c|c|}
\hline \multirow[t]{2}{*}{ Items } & \multirow[t]{2}{*}{$\boldsymbol{\beta}$} & \multirow[t]{2}{*}{ SE } & \multirow[t]{2}{*}{ Wald } & \multirow[t]{2}{*}{$P$} & \multirow[t]{2}{*}{ OR } & \multicolumn{2}{|l|}{$95 \% \mathrm{Cl}$} \\
\hline & & & & & & Lower & Upper \\
\hline miR-95-3p & -1.189 & 0.495 & 5.773 & $0.016^{*}$ & 0.305 & 0.116 & 0.803 \\
\hline miR-1246 & -0.738 & 0.575 & 1.645 & 0.200 & 0.478 & 0.155 & 1.477 \\
\hline miR-631 & 2.066 & 0.833 & 6.155 & $0.013^{*}$ & 7.890 & 1.543 & 40.344 \\
\hline
\end{tabular}

$\beta$ regression coefficient, $S E$ standard error, $O R$ odds ratio, $C l$ confidence interval.

${ }^{*} p<0.05$.

accuracy for distinguishing DN from the other groups $(A U C=$ 0.863). The levels of miR-95-3p, miR-185-5p, miR-1246, and miR631 were all statistically significant in the IgAN group and MN group compared with the DM group and DN group, while the levels of miR-185-5p and miR-631 were not statistically significant in the IgAN group and MN group. The diagnostic models we established had high diagnostic accuracy for distinguishing IgAN from the other groups $(A \cup C=0.859)$ and had moderate 

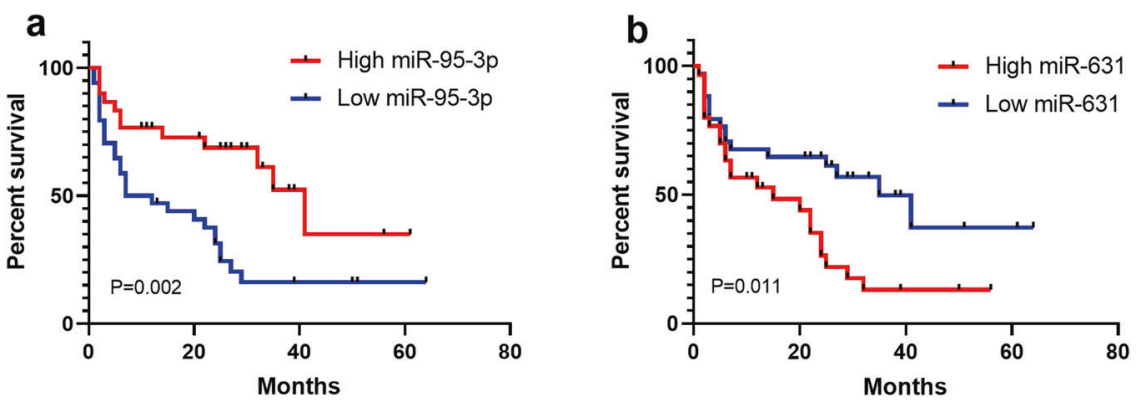

Fig. 4 Kaplan-Meier analysis of dialysis-free survival in patients with diabetic nephropathy. The subjects were dichotomized based on the mean of the covariates: a 0.822 for miR-95-3p; b 0.938 for miR-631. $P$ values refer to log-rank tests.

diagnostic accuracy for distinguishing $\mathrm{MN}$ from the other groups (AUC $=0.792$ ).

Many additional urine samples were collected to externally examine the accuracy of the diagnostic models in the validation cohort. The Model DM, which is based on two microRNAs (miR185-5p and miR-1246), exhibited high diagnostic accuracy (AUC = 0.928 ) in the validation cohort for distinguishing DM from the other groups. The Model DN, which is based on two microRNAs (miR-95-3p and miR-631), exhibited high diagnostic accuracy $(A U C=0.844)$ in the validation cohort for distinguishing DM from the other groups. Model lgAN, which is based on two microRNAs (miR-185-5p and miR-1246), demonstrated high diagnostic accuracy ( $A \cup C=0.849$ ) in the validation cohort for distinguishing IgAN from the other groups. However, Model MN, which is based on three microRNAs (miR-95-3p, miR-185-5p, and miR1246), demonstrated moderate diagnostic accuracy (AUC $=0.761$ ) in the validation cohort for distinguishing $\mathrm{MN}$ from the other groups.

We then explored the relationship between these microRNAs and disease severity and prognosis. We observed that the levels of miR-95-3p and miR-631 reflected the severity of DN. The Pearson correlation analysis revealed that the level of miR-95-3p was positively correlated with eGFR but was negatively correlated with the level of serum creatinine, classes of glomerular lesions, and scores of interstitial and vascular lesions. The level of miR-631 was positively correlated with proteinuria. Different severity levels of DN directly determine the judgment of the treatment effect and adjustment of the treatment plan. Therefore, the levels of miR-95$3 p$ and miR-631 could play an important role in clinical decisionmaking in patients with DN. In addition, our multivariate Cox regression analysis and Kaplan-Meier analysis also demonstrated that low levels of miR-95-3p and high levels of miR-631 increased the risk of progression to ESRD. Therefore, the levels of miR-95-3p and miR-631 could play an important role in the prognostic prediction of DN.

Urine contains many disease biomarkers that reflect an individual's health status and that are good indicators of kidney disease [33]. Recent studies have reported that many types of kidney diseases, such as primary lgAN, lupus nephritis, and minimal change nephropathy, could be detected by biomarkers in urinary sediment $[34,35]$. Duan et al. found that the levels of miR25-3p, miR-144-3p, and miR-486-5p were significantly higher in the IgAN group than in the normal control group [36]. Yan et al. found that urinary sediment could help with the differential diagnosis of lupus nephritis with endocapillary proliferative glomerulonephritis (EPGN) and IgAN with EPGN [35]. Compared with urine supernatant, urinary sediment was shown to be relatively less affected by humoral metabolic factors. Therefore, microRNAs in urinary sediment have the potential to serve as biomarkers for disease diagnosis and monitoring because they are relatively stable and are easily quantified. In addition, urinary sediment is obtained by a noninvasive method that can contribute to the early screening and monitoring of DN.
This study has a few limitations. First, our investigation did not refer to the mechanisms that cause alterations in microRNAs in patients with DN. Second, this was a single-center study, and it would be better if further multicenter studies and larger cohort studies are conducted for validation. Third, to further confirm the effect of microRNA levels on the prognosis of DN, it would be better to extend the follow-up time.

In conclusion, measurement of the levels of miR-95-3p, miR185-5p, miR-1246, and miR-631 could be a useful and noninvasive tool for distinguishing patients with DM, DN, and NDRD (IgAN and $M N)$. The levels of miR-95-3p and miR-631 can also reflect the severity and prognosis of DN.

\section{REFERENCES}

1. Cho NH, Shaw JE, Karuranga S, Huang Y, da Rocha Fernandes JD, Ohlrogge AW, et al. IDF Diabetes Atlas: Global estimates of diabetes prevalence for 2017 and projections for 2045. Diabetes Res Clin Pract. 2018;138:271-81.

2. Wanner C, Inzucchi SE, Lachin JM, Fitchett D, von Eynatten M, Mattheus M, et al. Empagliflozin and Progression of Kidney Disease in Type 2 Diabetes. N Engl J Med. 2016;375:323-34.

3. Arellano Buendía AS, Tostado González M, Sánchez Reyes O, García Arroyo FE, Argüello García R, Tapia E. et al. Immunomodulatory effects of the nutraceutical garlic derivative allicin in the progression of diabetic nephropathy. Int J Mol Sci. 2018;19:3107.

4. Han Q, Geng W, Zhang D, Cai G, Zhu H. ADIPOQ rs2241766 Gene Polymorphism and Predisposition to Diabetic Kidney Disease. J Diabetes Res. 2020;2020:5158497.

5. Li Y, Ren D, Shen Y, Zheng X, Xu G, Altered DNA. methylation of TRIM13 in diabetic nephropathy suppresses mesangial collagen synthesis by promoting ubiquitination of CHOP. EBioMedicine 2020;51:102582.

6. Burch E, Williams LT, Thalib L, Ball L. Short-term improvements in diet quality in people newly diagnosed with type 2 diabetes are associated with smoking status, physical activity and body mass index: the 3D case series study. Nutr Diabetes. 2020;10:25.

7. Zhou J, Chen X, Xie Y, Li J, Yamanaka N, Tong X. A differential diagnostic model of diabetic nephropathy and non-diabetic renal diseases. Nephrol Dial Transplant. 2008;23:1940-5.

8. Han Q, Zhu H, Chen X, Liu Z. Non-genetic mechanisms of diabetic nephropathy. Front Med. 2017;11:319-32.

9. Bermejo S, Soler MJ, Gimeno J, Barrios C, Rodríguez E, Mojal S, et al. Predictive factors for non-diabetic nephropathy in diabetic patients. Utility Ren biopsy Nefrologia. 2016;36:535-44.

10. Hou JH, Zhu HX, Zhou ML, Le WB, Zeng CH, Liang SS, et al. Changes in the spectrum of kidney diseases: an analysis of 40,759 biopsy-proven cases from 2003 to 2014 in China. Kidney Dis. 2018;4:10-19.

11. Liu S, Guo Q, Han H, Cui P, Liu X, Miao L, et al. Clinicopathological characteristics of non-diabetic renal disease in patients with type 2 diabetes mellitus in a northeastern Chinese medical center: a retrospective analysis of 273 cases. Int Urol Nephrol. 2016;48:1691-8.

12. Zhu H, Liu M, Yu H, Liu X, Zhong Y, Shu J, et al. Glycopatterns of urinary protein as new potential diagnosis indicators for diabetic nephropathy. J Diabetes Res. 2017;2017:5728087.

13. Li L, Zhang X, Li Z, Zhang R, Guo R, Yin Q, et al. Renal pathological implications in type 2 diabetes mellitus patients with renal involvement. J Diabetes Complications. 2017;31:114-21.

14. Liu MY, Chen XM, Sun XF, Zhou JH, Zhang XG, Zhu HY, et al. Validation of a differential diagnostic model of diabetic nephropathy and non-diabetic renal 
diseases and the establishment of a new diagnostic model. J Diabetes. 2014;6:519-26.

15. Chen Q, Zhu A, Wang J, Huan X. Comparative analysis of diabetic nephropathy and non-diabetic nephropathy disease. Saudi J Biol Sci. 2017;24:1815-17.

16. Huang F, Yang Q, Chen L, Tang S, Liu W, Yu X. Renal pathological change in patients with type 2 diabetes is not always diabetic nephropathy: a report of 52 cases. Clin Nephrol. 2007;67:293-7.

17. Parvex $P$. Are microRNA potential biomarkers in children with idiopathic nephrotic syndrome? EBioMedicine 2019;39:27-28.

18. Dewanjee S, Bhattacharjee N. MicroRNA: A new generation therapeutic target in diabetic nephropathy. Biochem Pharmacol. 2018;155:32-47.

19. Zhong F, Chen H, Xie Y, Azeloglu EU, Wei C, Zhang W, et al. Protein S protects against podocyte injury in diabetic nephropathy. J Am Soc Nephrol. 2018;29:1397-410.

20. Wang G, Lai FM, Lai KB, Chow KM, Li KT, Szeto CC. Messenger RNA expression of podocyte-associated molecules in the urinary sediment of patients with diabetic nephropathy. Nephron Clin Pract. 2007;106:c169-79.

21. Wang G, Kwan BC, Lai FM, Chow KM, Li PK, Szeto CC. Urinary sediment miRNA levels in adult nephrotic syndrome. Clin. Chim. Acta 2013;418:5-11.

22. Szeto CC, Ching-Ha KB, Ka-Bik L, Mac-Moune LF, Cheung-Lung CP, Gang W, et al. Micro-RNA expression in the urinary sediment of patients with chronic kidney diseases. Dis. Markers 2012;33:137-44.

23. Wang G, Szeto CC. Methods of microRNA quantification in urinary sediment. Methods Mol Biol. 2013;1024:211-20.

24. American Diabetes Association. Classification and diagnosis of diabetes: standards of medical care in diabetes-2019. Diabetes Care. 2019;42:13-S28.

25. Tervaert TW, Mooyaart AL, Amann K, Cohen AH, Cook HT, Drachenberg CB, et al. Pathologic classification of diabetic nephropathy. J Am Soc Nephrol. 2010;21:556-63.

26. Kdoqi. KDOQI clinical practice guidelines and clinical practice recommendations for diabetes and chronic kidney disease. Am J Kidney Dis. 2007;49:S12-154.

27. Lopez-Romero P. Pre-processing and differential expression analysis of Agilent microRNA arrays using the AgiMicroRna Bioconductor library. BMC Genomics. 2011;12:64.

28. Hsieh AR, Huang YC, Yang YF, Lin HJ, Lin JM, Chang YW. et al. Lack of association of genetic variants for diabetic retinopathy in Taiwanese patients with diabetic nephropathy. BMJ Open Diabetes Res Care. 2020;8:e000727.

29. Soleymanian T, Hamid G, Arefi M, Najafi I, Ganji MR, Amini M, et al. Non-diabetic renal disease with or without diabetic nephropathy in type 2 diabetes: clinical predictors and outcome. Ren Fail. 2015;37:572-5.

30. Matsushita M, Muramoto A, Nomura E, Eguchi Y, Kato A, Sano Y, et al. The Smart Life Stay (SLS) program: effects of a lifestyle intervention program in combination with health tourism and health guidance for type 2 diabetes. Nutr Diabetes. 2020;10:33.

31. Baker MA, Davis SJ, Liu P, Pan X, Williams AM, Iczkowski KA, et al. Tissue-specific microRNA expression patterns in four types of kidney disease. J Am Soc Nephrol. 2017;28:2985-92.

32. Szeto CC. Urine miRNA in nephrotic syndrome. Clin Chim Acta 2014;436:308-13.

33. Aitekenov S, Gaipov A, Bukasov R. Review: Detection and quantification of proteins in human urine. Talanta. 2021;223:121718.
34. Yuan M, Tan Y, Li J, Yu X, Zhang H, Zhao M. Urinary sediments could differentiate endocapillary proliferative lupus nephritis and endocapillary proliferative $\lg A$ nephropathy. Int Immunopharmacol. 2020;90:107122.

35. Gu W, Huang W, Zhang J, Qian S, Cao H, Ge L. Evaluation of urinary inflammatory index in rapid screening of urinary tract infection. Sci Rep. 2020;10:19306.

36. Duan ZY, Cai GY, Bu R, Lu Y, Hou K, Chen XM. Selection of urinary sediment miRNAs as specific biomarkers of IgA nephropathy. Sci Rep. 2016;6:23498.

\section{ACKNOWLEDGEMENTS}

This study was funded by the National Natural Science Foundation of China (No 61971441), the National Key R\&D Program of China (Nos. 2016YFC1305500, 2018YFA0108803). We thank Aimin Qi for her help.

\section{COMPETING INTERESTS}

The authors declare no competing interests.

\section{ADDITIONAL INFORMATION}

Supplementary information The online version contains supplementary material available at https://doi.org/10.1038/s41387-021-00166-z.

Correspondence and requests for materials should be addressed to D.Z. or H.Z.

Reprints and permission information is available at http://www.nature.com/ reprints

Publisher's note Springer Nature remains neutral with regard to jurisdictional claims in published maps and institutional affiliations.

Open Access This article is licensed under a Creative Commons Attribution 4.0 International License, which permits use, sharing, adaptation, distribution and reproduction in any medium or format, as long as you give appropriate credit to the original author(s) and the source, provide a link to the Creative Commons license, and indicate if changes were made. The images or other third party material in this article are included in the article's Creative Commons license, unless indicated otherwise in a credit line to the material. If material is not included in the article's Creative Commons license and your intended use is not permitted by statutory regulation or exceeds the permitted use, you will need to obtain permission directly from the copyright holder. To view a copy of this license, visit http://creativecommons. org/licenses/by/4.0/.

(c) The Author(s) 2021 